\title{
Smart Surveillance System using Raspberry Pi and Face Recognition
}

\author{
Chinmaya Kaundanya ${ }^{1}$, Omkar Pathak ${ }^{2}$, Akash Nalawade ${ }^{3}$, Sanket Parode ${ }^{4}$ \\ BE Student, Computer Engineering, MMCOE, Pune, India ${ }^{1,2,3,4}$
}

\begin{abstract}
This paper proposes the Smart Surveillance System using Raspberry Pi and Image Processing. This system will serve as smart security module for monitoring. Traditional surveillance systems only records the activities based on motion, but this system serves the purpose of facial recognition so as to reduce the error caused due to motion detection .Raspberry Pi camera module is used to capture images once the motion is detected by the PIR Sensor. This system will monitor when motion detected and checks for the faces in the image captured and with the help of face recognition alerts if the face detected is not stored in the database.
\end{abstract}

Keywords: Computer Vision, Local Binary Pattern, PIR Sensor, Raspberry Pi-Camera, Raspberry Pi.

\section{INTRODUCTION}

Surveillance is one of the important aspects in various fields such as banking sectors, military areas, or personal security. Due to exponential rise in burglary and theft activities, surveillance systems are proving to be a great source of security. Due to ever increasing technology people are relying on advanced technologies for their security purposes. Security systems such as CCTV have proven to be hugely popular for security purposes due to their cost efficient nature and easy maintenance. Surveillance is very helpful for law enforcement to investigate/prevent criminal activities, for recognizing and monitoring threats. Also, surveillance systems have always been playing a vital role in dealing with the burglary cases. These CCTV systems tend to monitor activities continuously. This results in high power consumption and memory wastage. Moreover, it does not give alert on any suspicious activities detected.

There are systems available other than CCTV such as Retina scanner, fingerprint scanner, IR lasers, RFID systems only with the drawback that they are cost inefficient with high implementation and maintenance costs. Hence, such systems are not a preferred way for security purposes for small scale applications. Proposed system covers all these drawbacks by its efficiency, portability. This surveillance system is low-cost and user-friendly too.

\section{LITERATURE SURVEY}

[1] Ms. Naga Jyoti and Mr. K. Vijaya Vardhan have proposed a system which captures the images and upload it to cloud server whenever a motion is detected. They also propose to send SMS alerts to users using GSM module.

[2] M. Surya Deekshith Gupta, Vamsikrishna Patchava, and Virginia Menezes have implemented a system which continuously captures the surroundings and if there is any moment, it turns on the light and captures the screesnshots that results in sending of those to authorised person as an alert.

[3] Aruni Singh, Sanjay Kumar Singh, Shrikant Tiwari have implemented comparison of various face recognition algorithms inlcuding eigenfaces, fisherfaces, Principle Component Analysis, Local binary Pattern. In holistic based algorithms PCA has range of accuracy from (51-72)\%, LDA (48.50-76.50)\%, iSVM (63.5-79)\% while texture based algorithm LBP shows the identification accuracy (60-94.5)\% and feature based algorithm SIFT demonstrates the accuracy range (61-94)\% at various image compression levels.

[4] Aamir Nizam Ansari, Mohamed Sedky, Neelam Sharma, Anurag Tyagi have implemented a system in which Raspberry Pi executes the processing of all the data and after the data is analyzed then the set actions are triggered for example sending an email on detection of motion and uploading images and videos to the ftp server.

[5] R.Chandana, Dr.S.A.K.Jilani, Mr.S.Javeed Hussain have proposed a system using Raspberry Pi and Gyroscope sensor. When a movement is detected, the Pi captures the image and then sends an alert email with the captured image. The sensor data is visualized in the form of charts on Thing Speak.

\section{III.PROPOSED METHODOLOGY}

The aim is to make a smart surveillance system using Raspberry Pi along with PIR sensor and Raspberry Pi-Camera. PIR sensor is used to detect the motion whenever someone comes within its range. As soon as PIR Sensor detects the motion, Pi-Camera activates and captures an image. This image is then stored in the system and finds for a human face in the captured image using OpenCV and Python. The detected human face is then compared with the faces stored in 
the database using Local Binary Pattern algorithm. If the face matches with the ones in the database no alert is generated else host gets a text message on his android mobile phone via Pushetta application using Wifi adapter. In this way, this system helps to identify only unauthorised persons. This helps to overcome the drawback of CCTV and Motion Detection systems which only monitor or alert host based on he motion detected whether it is authorised person or not.

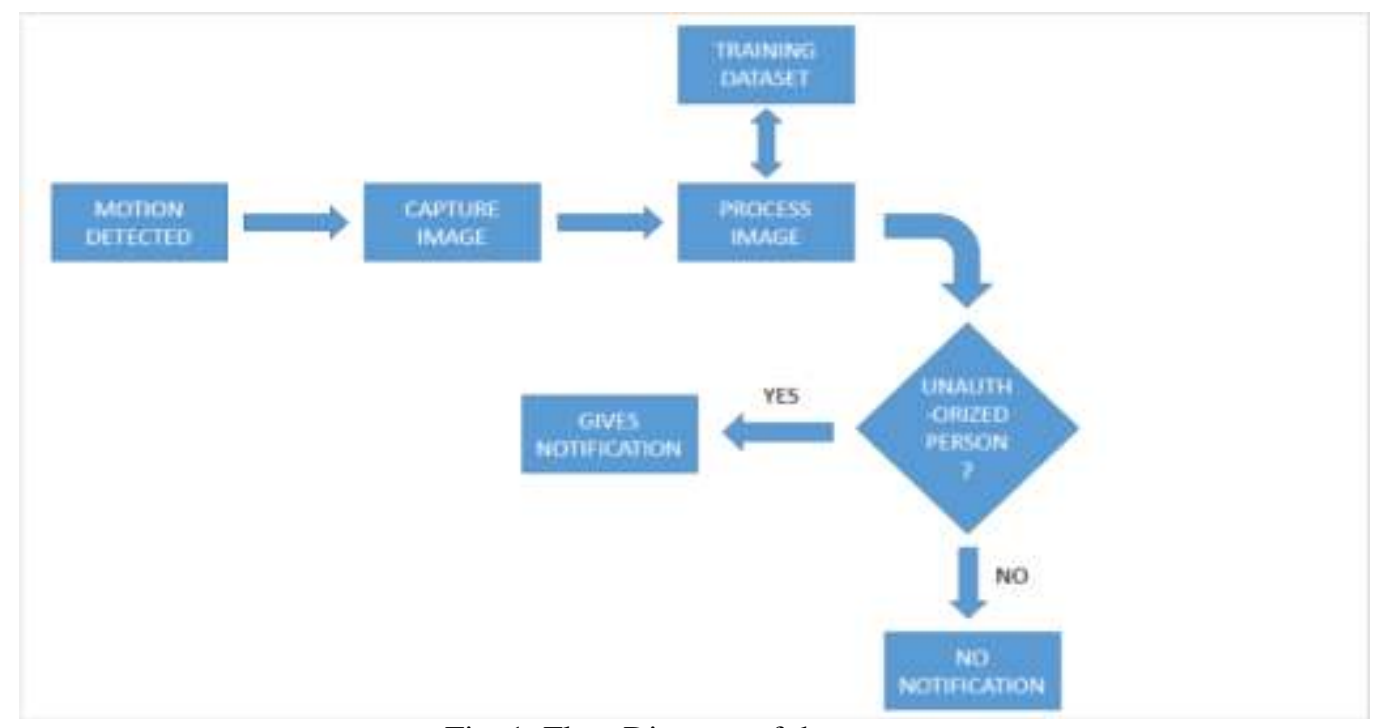

Fig. 1 Flow Diagram of the system

\section{IV.HARDWARE IMPLEMENTATION}

The system consists of Raspberry Pi, PIR Sensor, Raspberry Pi-Camera, Wifi adapter and Power Supply.

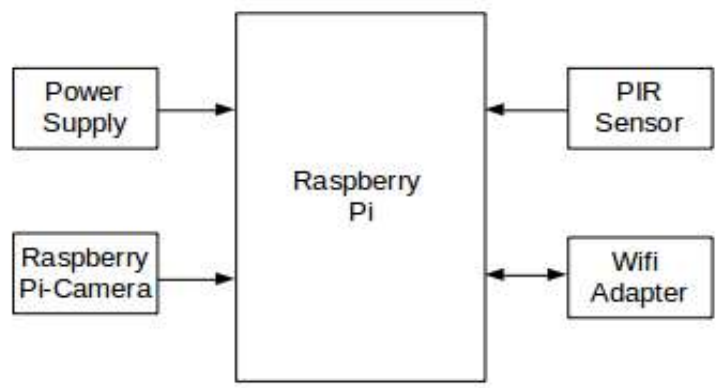

Fig. 2 Block Diagram

\section{A. Raspberry Pi}

Raspberry Pi is a low cost (35 dollar), credit card sized, computer that performs various applications. Some of its main features include 1GB of RAM, 4 USB Ports, General Purpose Input Output pins, Linux support. These features gives programmers a wide range for diverse applications.

\section{B. PIR Sensor}

Passive Infrared Sensor is an electronic sensor that measures infrared lights radiating from the objects in its field of view. Its maximum range is about $10 \mathrm{~m}$ which is suitable for motion detection applications.

\section{RaspberryPi-Camera}

Raspberry Pi-Camera module is a 8MP camera with full HD recording capability. This provides a perfect solution for face recognition.

\section{SOFTWARE IMPLEMENTATION}

This system is largely based on Python programming from detecting the motion to generating an alert. Various Python libraries are used to control PIR Sensor for detecting the motion, Python is used for Pi-Camera to capture and process 
images. The captured image is then processed using OpenCV library that integrates with Python. The Face-recognition part is carried out by the Local Binary Pattern(LBP) algorithm.

\section{A. OpenCV}

OpenCV (Open Computer Vision) is a library mainly aimed at real-time computer vision. It provides great support for face detection and face-recognition techniques using Python.

\section{B. Local Binary Patterns}

Local binary patterns (LBP) is a type of algorithm used for classification in computer vision. It has been found to be a powerful feature extraction and classification purposes.

\begin{tabular}{|c|c|c|c|c|c|c|}
\hline 5 & 9 & 1 & \multirow{3}{*}{ Thresholding } & 1 & 1 & $\mathrm{O}$ \\
\hline 4 & 4 & 6 & & 1 & & 1 \\
\hline 7 & 2 & 3 & & 1 & O & $\mathrm{O}$ \\
\hline
\end{tabular}

Binary: 11010011

Decima $1: 211$

Fig. 3 Intensity calcuations in Local Binary Pattern

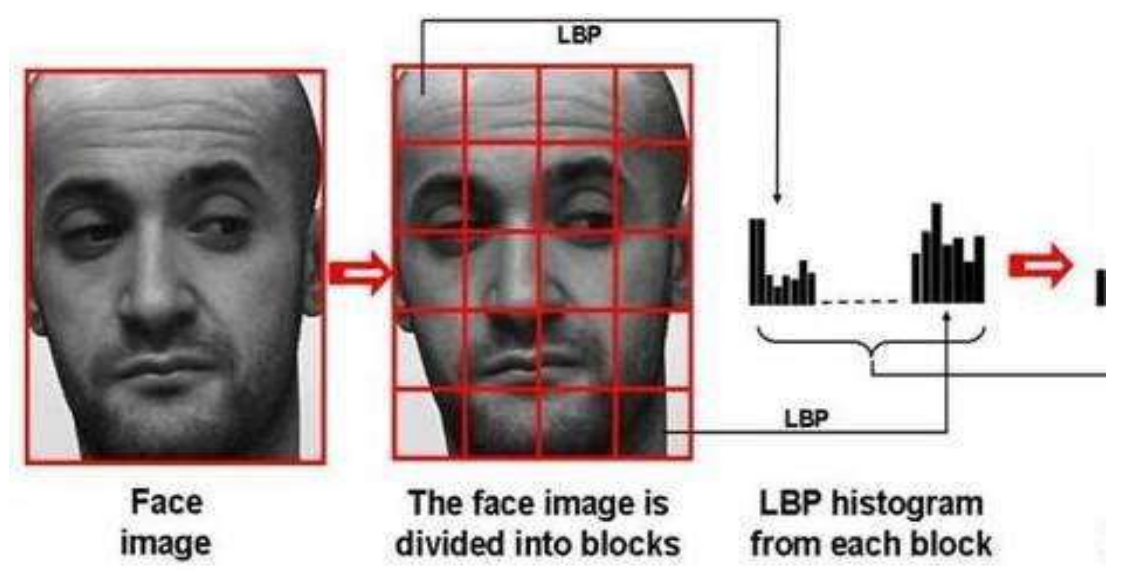

Fig. 4 Local Binary Pattern algorithm working

VI.EXPERIMENTAL RESULTS

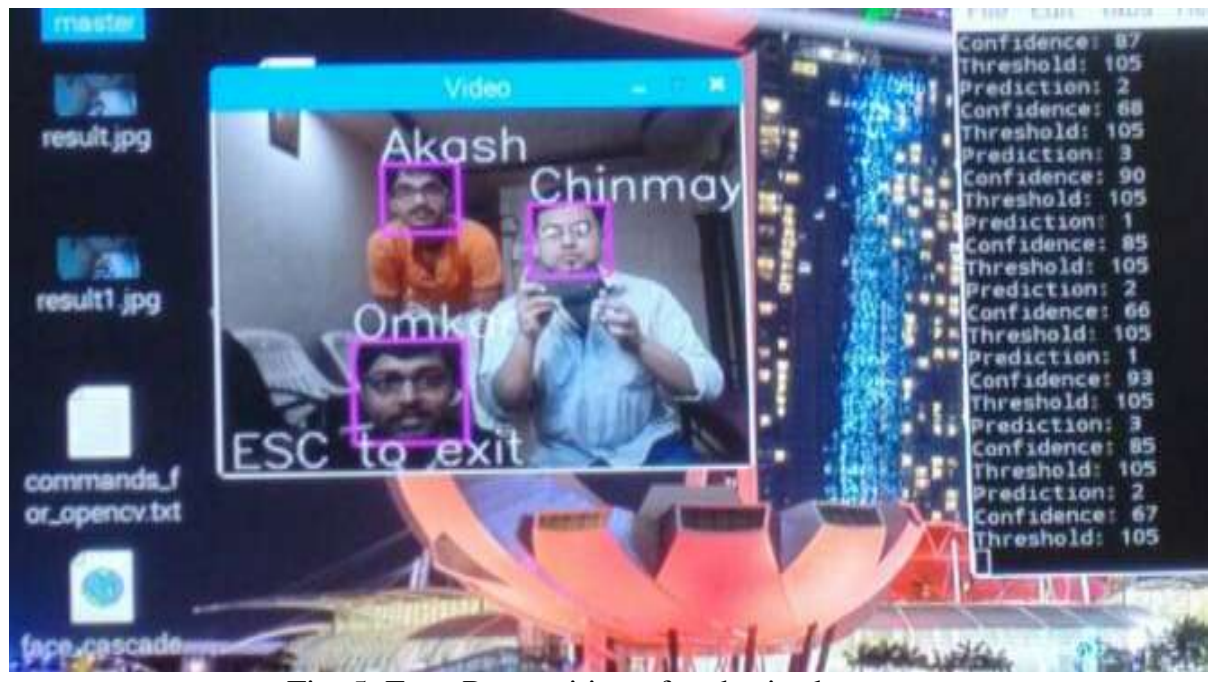

Fig. 5 Face-Recognition of authorised person 
Lots of experiments have been carried out to get the range of PIR Sensor accurately. For best results of facerecognition, large number of images of authorized persons have been taken as datasets for comparison of images to determine authorized and unauthorized persons. About 70 to 80 images of per person with their different facial expressions have been taken into consideration while performing the tests. Accuracy for the face-recognition is about $80 \%$. Different images have to be stored in different situations and light intensities such as light and dark surroundings for obtaining best results.

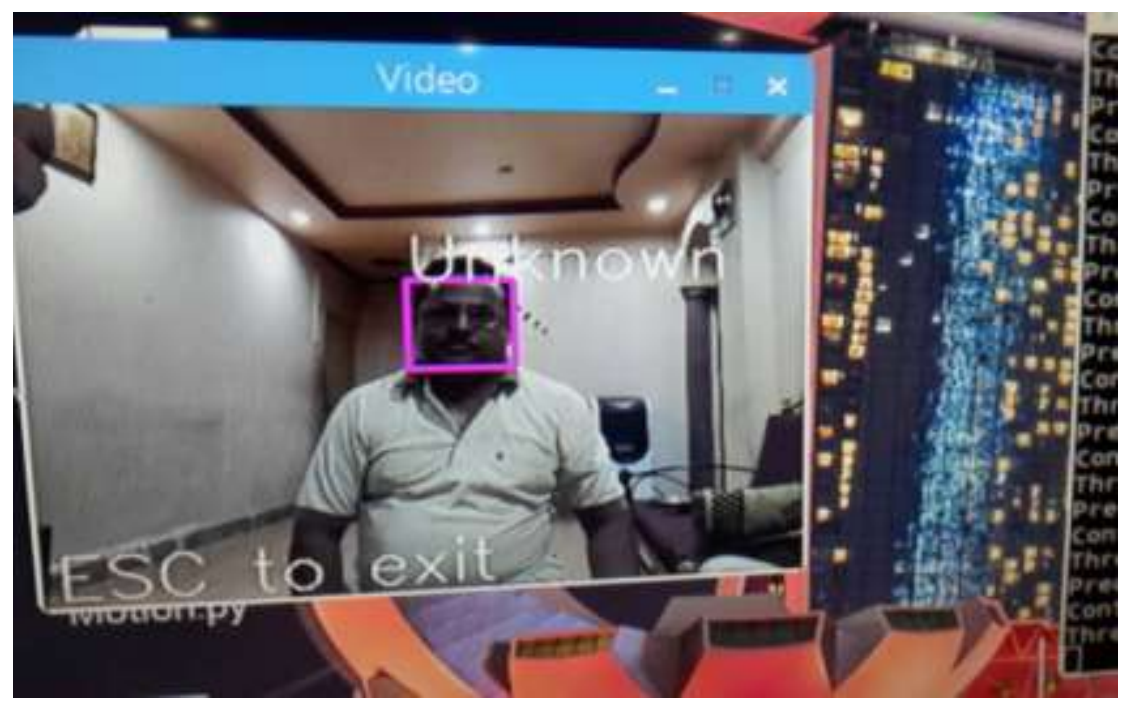

Fig. 6 Face-Recognition of Unauthorised person

Fig. 5 shows once a motion is detected the captured image is processed and categorized into authorized and unauthorized persons. Authorized persons are detected as shown above.

Fig. 6 shows how unauthorized person is detected by the system. This generates an alert and notifies user on his android pushetta application.

\section{CONCLUSION}

Surveillance system provides an efficient way for monitoring suspicious activities. Traditional systems are efficient and have low maintenance cost. However, energy consumption is more as the system is continuously powered on. Proposed IOT based smart surveillance system provides energy management by turning the system ON, based on the occurrence of a particular motion. System will sense the motion and depending on the detected motion system will switch on the camera, capture the image of intruder, recognize it and send a notification on owner's smart phone if the person is not recognized by the system.

\section{REFERENCES}

[1] Ms. Naga Jyoti and Mr. K. Vijaya Vardhan, "Design And Implementation Of Real Time Security Surveillance System Using IoT", Communication and Electronics Systems (ICCES), International Conference: IEEE, 2016.

[2] M. Surya Deekshith Gupta, Vamsikrishna Patchava, and Virginia Menezes: "Surveillance and Monitoring System Using Raspberry Pi and SimpleCV": Green Computing and Internet of Things (ICGCIoT), IEEE, 2016.

[3] Aruni Singh, Sanjay Kumar Singh, Shrikant Tiwari, "Comparison of Face Recognition Algorithms on Dummy Faces", The International Journal of Multimedia \& Its Applications (IJMA) Vol.4, No.4, August 2012.

[4] Aamir Nizam Ansari, Mohamed Sedky, Neelam Sharma, Anurag Tyagi, “An Internet of Things Approach for Motion Detection using Raspberry Pi”, International Conference on Intelligent Computing and Internet of Things (IC1T), 2015.

[5] R.Chandana, Dr.S.A.K.Jilani, Mr.S.Javeed Hussain, "Smart Surveillance System using Thing Speak and Raspberry Pi", International Journal of Advanced Research in Computer and Communication Engineering Vol. 4, Issue 7, July 2015.

[6] Sanjana Prasad, P.Mahalakshmi, A.John Clement Sunder, R.Swathi, "Smart Surveillance Monitoring System Using Raspberry PI and PIR Sensor", (IJCSIT) International Journal of Computer Science and Information Technologies, 2014.

[7] Huu-Quoc Nguyen, Ton Thi Kim Loan, Bui Dinh Mao, Eui-Nam Huh, "Low cost real-time system monitoring using Raspberry Pi", IEEE, 2015

[8] Sarabjit Singh, Amritpal Kaur, T aqdir, "A Face Recognition Technique using Local Binary Pattern Method", International Journal of Advanced Research in Computer and Communication Engineering, 2015.

[9] Sushma Jaiswal, Dr. (Smt.) Sarita Singh Bhadauria , Dr. Rakesh Singh Jadon, “ Comparison between face recognition algorithm-eigenfaces, fisherfaces and elastic bunch graph matching ", Journal of Global Research in Computer Science, 2011

[10] https://en.wikipedia.org/wiki//mage_processing

[11] https://en.wikipedia.org/wiki/Local_binary_patterns 